\title{
Searching in 2-Dimensional mass space for final states with 2 invisible particles
}

\section{G. Anagnostou}

Institute of Nuclear and Particle Physics, NCSR Demokritos,

Patr. Gregoriou E and 27 Neapoleos Str, 15341 Agia Paraskevi, Greece

E-mail: georgios.anagnostou@cern.ch

ABSTRACT: A method to search for particles of unknown masses in final states with two invisible particles is presented. Searching for final states with missing energy is a challenging task usually performed in the tail of a missing energy related distribution. The search method proposed is based on a 2-Dimensional mass reconstruction of the final state with two invisible particles. Thus, a bump hunting is possible, allowing a stronger signal versus background discrimination. Parameters of the new theory can be extracted from the mass distributions, a valuable step towards understanding its true nature. The proof of principle is based on the existing SM top pairs in their dilepton final state. The method is applicable in many interesting searches at the LHC, including dark matter candidates or heavy top partners.

KeYWORDS: Supersymmetry Phenomenology

ArXiv EPrint: 2009.10032 


\section{Contents}

1 Introduction 1

2 Event simulation and selection $\quad 3$

3 The method 4

4 2D mass reconstruction - example topologies $\quad 8$

4.1 Top pairs 8

4.2 Search for anything decaying like dilepton top pairs 9

$\begin{array}{ll}4.3 \text { Search for stop pairs } & 10\end{array}$

$\begin{array}{ll}4.4 \text { Top pair identification/other topologies } & 12\end{array}$

5 Mass measurements $\quad 12$

6 Conclusions 14

A System of equations for top pairs in the dilepton channel 16

\section{Introduction}

After Higgs boson discovery, it is not easy to figure out which experimental signatures are the most promising. Missing energy final states are an interesting case, predicted by well motivated theories beyond the Standard Model (BSM) [1-3]. The energy missing originates from invisible particles such as neutrinos (e.g heavy top partners) or weakly interacting massive particles (WIMPs) as dark matter candidates.

In the case of Higgs boson, the Standard Model (SM) was predicting everything except its mass: the cross-section, its decay channels, the couplings to other particles etc. Even the Higgs mass was predicted indirectly by the electroweak fit, so that the search could be narrowed to a few tens of GeV of the invariant mass spectrum [4]. The LHC machine and detectors were actually designed for Higgs boson discovery. Based on accurate Monte-Carlo predictions the search could be tuned to extract the signal easier. For example, multivariate methods were trained to discriminate signal from background processes based on simulated Higgs events. Without this tuning in the design of the experiment/detectors/analysis, Higgs discovery would probably be harder to be established and have taken longer time. In addition, the discovery was based on invariant mass observables, in channels with visible decay products (photons, electron/muons). It was a bump hunt, a case where the shape of signal and background processes is different, so that a robust discovery is easier.

Searching for new physics in final states with missing energy is a challenging task and certainly much harder than searching for a particle with known properties such as the SM 

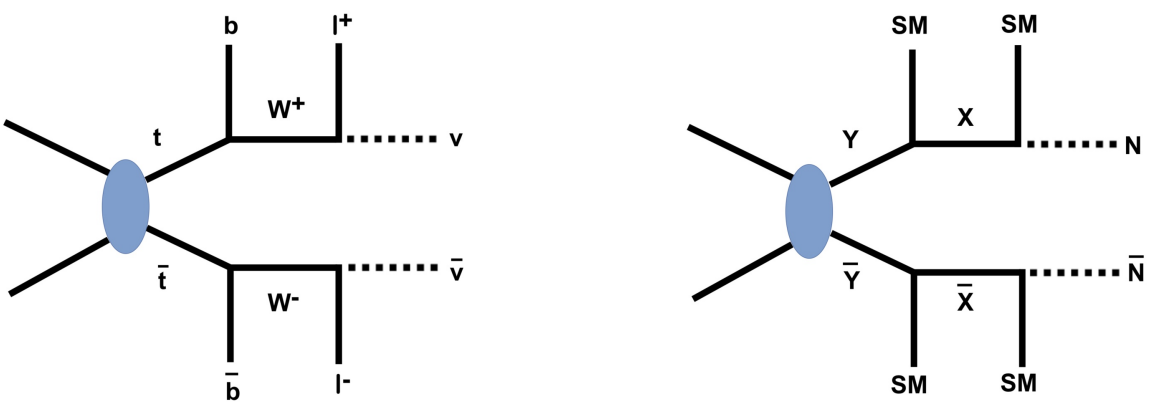

Figure 1. The existing SM top pair decay with two invisible particles (left) and a similar pair produced generic BSM topology (right).

Higgs boson [5, 6]. An existing SM diagram with two invisible particles can be seen in figure 1 together with a similar pair produced generic BSM topology. Understanding the physics is much easier in possible BSM signals with visible particles. In this case, the invariant masses can be reconstructed up to combinatoric ambiguities. In final states with invisible particles, instead of a bump hunt in a small region of the invariant mass spectrum, the search has to be performed using the tail of a missing energy related distribution. Due to the small difference in shape between signal and background distributions the establishment of a discovery in this case is a difficult problem. In addition, any mismeasurement or mismodelling of any type of the physics objects used can introduce a fake missing energy signal. Even when a discovery is established, it is likely that only limited additional information about the new physics would be available.

The classical way to search for new particles is by applying a set of selection rules (cuts) which are usually targeting the region of phase space where signal dominates. This depends strongly on the specific model and usually selects rectangular phase space regions of the energy and transverse momenta of the reconstructed objects. Sometimes these kinematic observables are used as input to multivariate discriminators like likelihoods, boosted decision trees and neural networks. It is common that experiments tune their cuts/multivariate observables such as to be optimized/trained for a specific choice of the BSM model parameters. If new physics is not in the parameter space region for which the search was optimized/trained, it is likely that the sensitivity of the search will be reduced. So it is highly desirable that the performance of the search does not depend on the model or that it is as model independent as possible.

The mass and spin are the most important characteristics of elementary particles according to quantum field theory. The mass space is ideal for resonance searches: events are concentrated in a small region, whereas background events have no reason to do the same. Mass observables do not require optimization or training. Ideally, searches should be performed in multidimensional mass spaces with as many dimensions as the number of unknown particles. They are commonly used in searches with visible particles and are also used in topologies with a single invisible particle. This paper proposes to use them extensively in final states with two invisible particles, as there are many interesting applicable topologies which can benefit from the advantages of mass observables. 

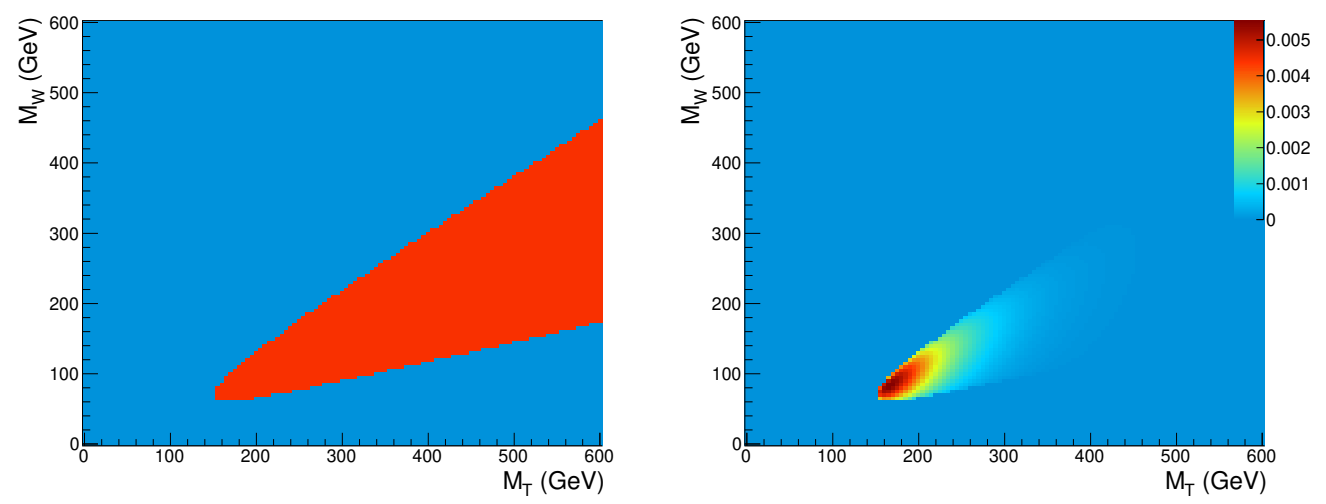

Figure 2. (Left): solvability for one of the possible solutions of a single top-pair event in the 2-Dimensional mass plane. (Right): solvability weighted with the PDFs and normalized to unit volume for the same solution and event.

In the next sections, the method to perform 2-Dimensional mass reconstruction in final states with two invisible particles is described. Initially, the generation and simulation of the signal and background processes is discussed in section 2 . The next section concerns the description of the method. Section 4 has several topologies and applications starting with the dilepton top pairs as a proof of principle. Then, in the next example, a generic topology of anything decaying like dilepton top pairs is presented ( $\left.\mathrm{pp} \rightarrow \mathrm{T}^{\prime} \overline{\mathrm{T}}^{\prime} \rightarrow \mathrm{W}^{\prime} \mathrm{bW} \mathrm{W}^{\prime} \mathrm{b}\right)$. The most interesting application concerning dark matter searches at the LHC follows: the search for a pair produced stop quark decaying to charginos and bquarks ( $\mathrm{pp} \rightarrow \tilde{\mathrm{t}} \overline{\mathrm{t}} \rightarrow \mathrm{x}_{1}^{+} \mathrm{bx}{ }_{1}^{-} \overline{\mathrm{b}}$ ), with charginos decaying further to leptons and sneutrinos. This is the most general case as the invisible particles are massive. The 2-Dimensional mass reconstruction allows these searches to be performed as bump hunting with a single entry per event, with all the advantages offered in terms of discovery. More applications are discussed such as the usage of the 2-Dimensional mass reconstruction for identification of dilepton top pairs and the application of the method in cases with a single unknown mass.

Finally, in section 5, the prospects of measuring the masses of top quark or possible new discovered particles is discussed. The description that follows is based on simulated events with all the complexity available in a fast simulation package. It is worth mentioning that the method has already been applied in CMS Run1 dataset for a generic search for anything decaying like dilepton top pairs [7-10].

\section{Event simulation and selection}

All signal and background processes were generated and simulated for an intergrated luminosity of $50 \mathrm{fb}^{-1}$ at an LHC collision energy of $13 \mathrm{TeV}$. The SM processes were generated using Pythia8 [12]: top pairs, single boson (Drell-Yan, W+jets), dibosons (WW, WZ, ZZ) as well as single top events.

For the generic search of anything decaying like top pairs $\left(\mathrm{pp} \rightarrow \mathrm{T}^{\prime} \overline{\mathrm{T}}^{\prime} \rightarrow \mathrm{W}^{\prime} \mathrm{b} \mathrm{W}^{\prime} \mathrm{b}\right)$ signal events were generated using the littlest Higgs Model $[13,14]$. An implementation of the latter can be found in Whizard 2.2.0 event generator $[15,16]$. The model has both a pair 


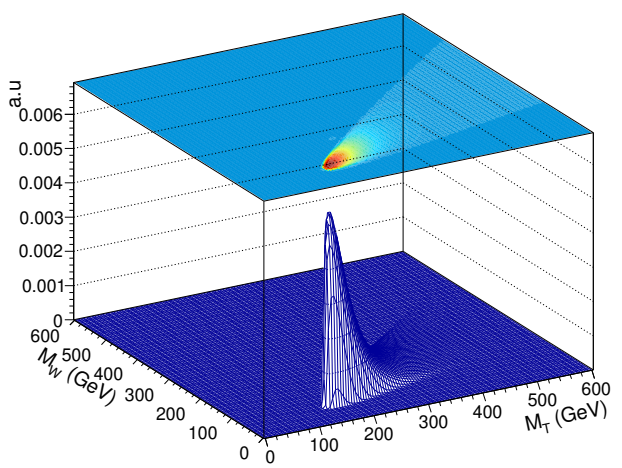

Figure 3. Average PDF weighted solvability for the 100 smeared events produced from the same initial top pair event. The distribution is normalized to unit volume.

produced heavy top partner $\mathrm{T}^{\prime}$ as well as a new heavy gauge boson $\mathrm{W}^{\prime}$. In order to create a simplified model, initially the hard scattering $\left(\mathrm{pp} \rightarrow \mathrm{T}^{\prime} \overline{\mathrm{T}}^{\prime}\right)$ was generated with Whizard. In this first step, $\mathrm{T}^{\prime}$ was produced as a stable particle (zero width). In the next simulation step, the decays of both heavy top partner $\left(\mathrm{T}^{\prime} \rightarrow \mathrm{W}^{\prime} \mathrm{b}\right)$ and new heavy gauge boson to leptons ( $\mathrm{W}^{\prime} \rightarrow 1, \nu, 1=\mathrm{e}, \mu$ ) were performed with Pythia8 using LHA interface [17].

The supersymmetric search for stop quarks $\left(\mathrm{pp} \rightarrow \tilde{\tilde{\mathrm{t}}} \rightarrow \mathrm{x}_{1}^{+} \mathrm{bx}_{1}^{-} \overline{\mathrm{b}} \rightarrow \mathrm{bl}^{+} \tilde{\nu} \overline{\mathrm{bl}}{ }^{-} \overline{\tilde{\nu}}\right)$ is performed with events generated with Madgraph5 [18]. The pair produced stop quarks decay to charginos and $\mathrm{b}$ quarks with the charginos decaying further to leptons (electrons or muons) and sneutrinos. The decay of sneutrino to the invisibles neutrino and neutralino $\left(\tilde{\nu} \rightarrow \chi_{1}^{0} \nu\right)$ is also taking place in order to have missing energy from stable particles for the next steps. The hadronization is performed with Pythia8 before the events are used as an input to the detector simulation.

The events yields in the simulated data samples are normalized to the corresponding integrated luminosity of $50 \mathrm{fb}^{-1}$ according to their theoretical cross sections. The latter, together with the number of simulated events can be seen in tables $1-2$. For $\mathrm{W}+$ jets and $\mathrm{Z}+$ jets, these are taken from next-to-next-to-leading-order (NNLO) calculations [19]. For the dibosons WZ/ZZ as well for WW the NLO [20] and NNLO [21] calculations are used respectively. For the simulated $t \overline{\mathrm{t}}$ sample, the full NNLO+NNLL calculation [22] - [27] performed with the TOP ++2.0 program [28] is used, assuming a top quark mass value of $172.5 \mathrm{GeV}$. For the signals as well as for the single top background processes the cross-section is estimated by the corresponding generator. All signal and background processes were further processed with Delphes-3.4.0 detector simulation package [29] using the parameters of a typical LHC detector (CMS).

\section{The method}

The dilepton top pairs system of equations has an analytical solution [30, 31]. The algorithm takes as an input the masses of top quark and W boson as well as the visible particle's momenta and gives as an output the momenta of the two neutrinos. For any $\mathrm{M}_{\mathrm{T}}$ and $\mathrm{M}_{\mathrm{W}}$ 


\begin{tabular}{|l|l|l|r|r|}
\hline Background MC samples & group & Generator & $\sigma[\mathrm{pb}]$ & \# Events \\
\hline $\mathrm{t} \overline{\mathrm{t}}$ & top pairs & Pythia.8 & 831.76 & 89310000 \\
\hline Drell $-\mathrm{Yan}, \mathrm{Z} \rightarrow l l, l=\mathrm{e}, \mu, \tau, 10 \mathrm{GeV}<\mathrm{M}_{l l}<50 \mathrm{GeV}$ & single boson & Pythia.8 & 22635 & 39800000 \\
\hline Drell $-\mathrm{Yan}, \mathrm{Z} \rightarrow l l, l=\mathrm{e}, \mu, \tau, \mathrm{M}_{l l}>50 \mathrm{GeV}$ & single boson & Pythia.8 & 6077.22 & 39760000 \\
\hline $\mathrm{W}+$ jets, $\mathrm{W} \rightarrow l \nu, l=\mathrm{e}, \mu$ & single boson & Pythia.8 & 41017.8 & 39920000 \\
\hline $\mathrm{WW}$ & diboson & Pythia.8 & 118.7 & 1085000 \\
\hline $\mathrm{WZ}$ & diboson & Pythia.8 & 47.13 & 1250000 \\
\hline $\mathrm{ZZ}$ & diboson & Pythia.8 & 16.52 & 1250000 \\
\hline s-channel single top & single top & Pythia.8 & 7.67 & 9760000 \\
\hline t-channel single top & single top & Pythia.8 & 159.0 & 9480000 \\
\hline
\end{tabular}

Table 1. Simulated samples of the background processes with their corresponding cross section and produced event numbers.

\begin{tabular}{|l|l|l|r|r|}
\hline Signal MC samples & BSM Model & Generator & $\sigma[\mathrm{pb}]$ & \# Events \\
\hline $\mathrm{M}_{\mathrm{T}^{\prime}}=600 \mathrm{GeV} \mathrm{M}_{\mathrm{W}^{\prime}}=400 \mathrm{GeV}$ & Littlest Higgs & Whizard2.2 & 0.648 & 50000 \\
\hline $\mathrm{M}_{\mathrm{T}^{\prime}}=800 \mathrm{GeV} \mathrm{M}_{\mathrm{W}^{\prime}}=400 \mathrm{GeV}$ & Littlest Higgs & Whizard2.2 & 0.07 & 50000 \\
\hline $\mathrm{M}_{\mathrm{T}^{\prime}}=800 \mathrm{GeV} \mathrm{M} \mathrm{W}^{\prime}=600 \mathrm{GeV}$ & Littlest Higgs & Whizard2.2 & 0.07 & 50000 \\
\hline $\mathrm{M}_{\mathrm{T}^{\prime}}=1000 \mathrm{GeV} \mathrm{M} \mathrm{W}^{\prime}=400 \mathrm{GeV}$ & Littlest Higgs & Whizard2.2 & 0.015 & 50000 \\
\hline $\mathrm{M}_{\tilde{\mathrm{t}}}=1500 \mathrm{GeV} \mathrm{Mx}$ \\
\hline $\mathrm{M}_{\tilde{\mathrm{t}}}=1500 \mathrm{GeV} \mathrm{Mx}$ \\
\hline
\end{tabular}

Table 2. Simulated samples of the BSM signals with their corresponding cross section and produced event numbers.

values for which the system is solvable, all event kinematics are calculable, including the fractions of the proton's energies participating in the hard process. Using these fractions as an input to the Parton Distribution Functions (PDFs), a probability-score can then be assigned for each point of the 2-Dimensional mass plane. The one that is more likely to originate from a p-p collision can be chosen as the $\mathrm{M}_{\mathrm{T}}, \mathrm{M}_{\mathrm{W}}$ estimation for the event.

More specifically, the kinematics of $t \overline{\mathrm{t}}$ dilepton events can be expressed by two linear and six non linear equations (appendix). The system is solvable with respect to the unknown neutrino and antineutrino momenta, provided that the masses $\mathrm{M}_{\mathrm{T}}, \mathrm{M}_{\mathrm{W}}$, the momentum of bquarks and leptons as well as the missing energy components are available. Each possible input can give 0, 2 or four different solutions for the unknown neutrino and antineutrino momentum components. In addition, there are two possible combinations of bjets and leptons that could originate from the same top quark, giving in total up to eight solutions for specific $\mathrm{M}_{\mathrm{T}}$ and $\mathrm{M}_{\mathrm{W}}$ values. Knowledge of the momenta of the invisible neutrinos allows full kinematic reconstruction of the event including the four-vectors of $\mathrm{W}$ bosons, top quarks and the $\bar{t} \bar{t}$ system.

Searching for topologies with two invisible particles requires no a-priori knowledge of the masses, as this should be the result rather than the input. It is the inverse problem with respect to the analytical solution: given the visible particles and the topology we are looking for the unknown masses per event. In order to solve the inverse problem, every 


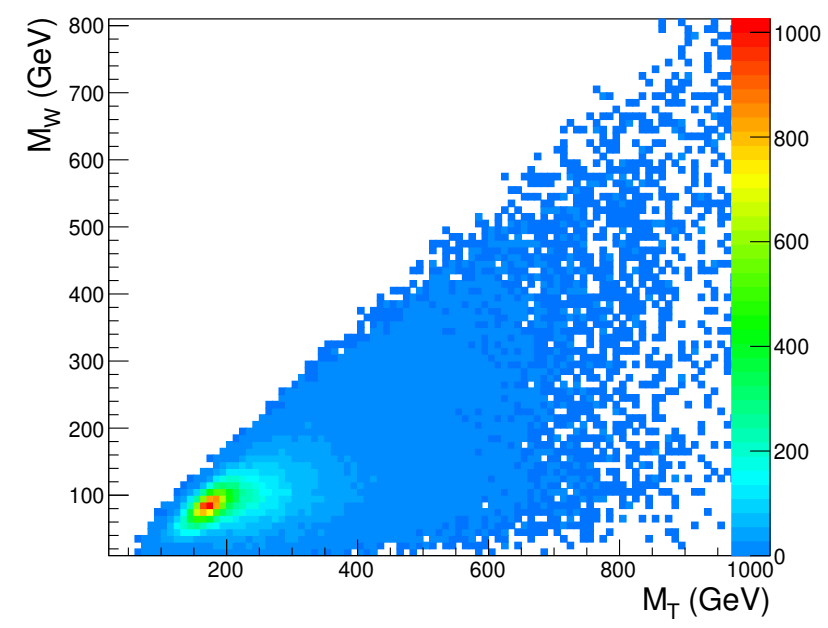

Figure 4. Reconstructed $\mathrm{M}_{\mathrm{T}}$ and $\mathrm{M}_{\mathrm{W}}$ per event for a sample of signal (top-pairs) and background events corresponding to an intergrated luminosity of $50 \mathrm{fb}^{-1}$ at $13 \mathrm{TeV}$.

point of the $\mathrm{M}_{\mathrm{T}}, \mathrm{M}_{\mathrm{W}}$ plane is tested for possible solutions. The mass plane can be scanned in steps of a few $\mathrm{GeV}$ to produce the area in which each one of the eight possible solutions exists or not. The step has been chosen to be $5 \mathrm{GeV}$ for all cases presented. The existance of a real solution makes the event solvable for this specific mass point. An example of such a solution area for one of its eight possible solutions of a single top pair event is plotted in figure 2 (left). The area provides a boundary in the lower mass region for the possible masses of top quark and $\mathrm{W}$ boson, as below these masses the event is not solvable.

Due to the finite collission energy there is also an upper limit to the allowed masses produced. The center of mass energy of the partons partipipating in the hard scattering has to be smaller than the LHC collision energy. For a p-p collider this energy limit can be expressed fully by the parton distribution functions (PDFs) of the proton in the following way: each solution allows full reconstruction of event kinematics, including the estimation of the energy $\mathrm{E}$ and $\mathrm{pZ}$ momenta component of the $\mathrm{t} \overline{\mathrm{t}}$ system. These variables can be easily transformed to the fraction of beam energy of the two partons participating in the hard scattering $\left(\mathrm{x}_{1,2}=\left(\mathrm{E} \pm \mathrm{p}_{\mathrm{Z}}\right) / \sqrt{\mathrm{s}}, \sqrt{\mathrm{s}}\right.$ being the center of mass energy). So each parton with fraction $\mathrm{x}_{\mathrm{i}}$ can be assigned with a probability $\mathrm{F}\left(\mathrm{x}_{\mathrm{i}}\right)$ to originate from a proton-proton collision. By multiplying the probabilities of the two incoming partons, a weight per mass point can be assigned for each solution. As there are more than one possible leading order parton-parton interactions ( $u \bar{u}, \bar{u} u, d \bar{d}, \bar{d} d, g g$ ), the weights from all possible combinations are summed to estimate a final event weight per solution and mass point. The weight can be written as $\sum_{\mathrm{a}, \mathrm{b}} \mathrm{F}^{\mathrm{a}}\left(\mathrm{x}_{1}, \mathrm{Q}\right) \mathrm{F}^{\mathrm{b}}\left(\mathrm{x}_{2}, \mathrm{Q}\right)$, where the summation is over all possible parton combinations, $\mathrm{F}^{\mathrm{a} / \mathrm{b}}(\mathrm{x}, \mathrm{Q})$ refers to the corresponding parton CT10 PDF set [32] and $\mathrm{Q}$ is the factorization scale set to $\mathrm{M}_{\mathrm{T}}$ 's value. For the estimation of the PDFs the LHAPDF-6.1.2 interface was used [33].

The PDF weight normalized to unit volume provides an upper bound for the mass values of both $\mathrm{M}_{\mathrm{T}}$ and $\mathrm{M}_{\mathrm{W}}$. The solution area shown in figure 2 (left) is weighted by the 

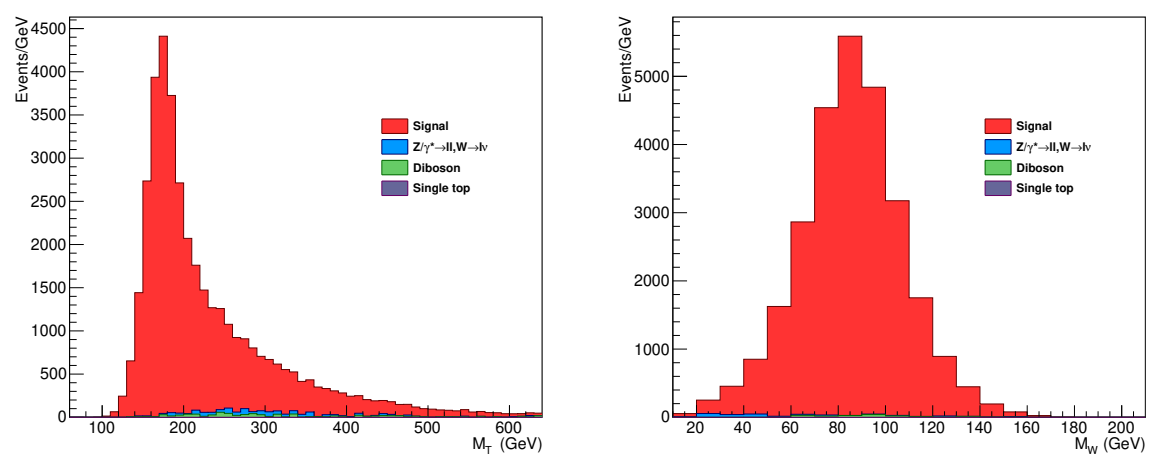

Figure 5. Reconstructed $\mathrm{M}_{\mathrm{T}}$ (left) as well as $\mathrm{M}_{\mathrm{W}}$ (right), for a sample of signal (top pairs) and background events corresponding to an intergrated luminosity of $50 \mathrm{fb}^{-1}$ at $13 \mathrm{TeV}$.

PDFs and the result is plotted in figure 2 (right). Each of the possible event solutions can produce such a distribution, so the maximum point of all distributions is the most likely to originate from a proton-proton collision and is therefore taken as the $\mathrm{M}_{T}$ and $\mathrm{M}_{\mathrm{W}}$ for this event. It is interesting to mention that the prefered mass point is not the one with the lowest $\mathrm{M}_{\mathrm{T}}$ and $\mathrm{M}_{\mathrm{W}}$ values as one might have guessed from the fact that PDFs favour lower mass values. Use of the solvability together with a matrix element weight which depends on the model has been proposed for top quark mass estimation in a single mass dimension [34-36]. This proposal has evolved to the matrix element weighting top mass measurement method in Tevatron [37], which has also been used at the LHC [38]. The proposal in this paper is to use the PDF weight to search for final states with two invisible particles in the 2-Dimensional mass plane of the unknown particles. No matrix elements are used so that the search is as model independent as possible.

Detector effects can change the momenta of the leptons and jets making a solvable event not-solvable. In many cases solvability can be recovered by smearing the leptons and jets according to detector resolution. For each initial event, N smeared events can be created by smearing the leptons and jets of the recorded event according to the detector resolutions. For each of these smeared events, the same procedure as described above is followed: the solution area is weighted by the PDFs. The result for each solution is averaged over all $\mathrm{N}$ smeared events to form the final observable by the formula $\sum_{\mathrm{i}=1}^{\mathrm{N}} \sum_{\mathrm{a}, \mathrm{b}} \mathrm{F}^{\mathrm{a}}\left(\mathrm{x}_{1}^{\mathrm{i}}, \mathrm{Q}\right) \mathrm{F}^{\mathrm{b}}\left(\mathrm{x}_{2}^{\mathrm{i}}, \mathrm{Q}\right)$, normalized to unit volume. An example is given in figure 3 for the same solution of the initial top pair event. Again, among all solutions, the one with the maximum PDF weight is chosen as the final $\mathrm{M}_{\mathrm{T}}$ and $\mathrm{M}_{\mathrm{W}}$ estimation for this event. The above procedure gives a single entry per event for each of the unknown masses. Is is worth emphasizing that combinations/solutions have different probabilities to originate from a proton-proton collision and the parton distribution functions can distinguish the highest one. This might be applicable to other cases with combinatoric backgrounds such as reconstruction of chains with visible particles. 

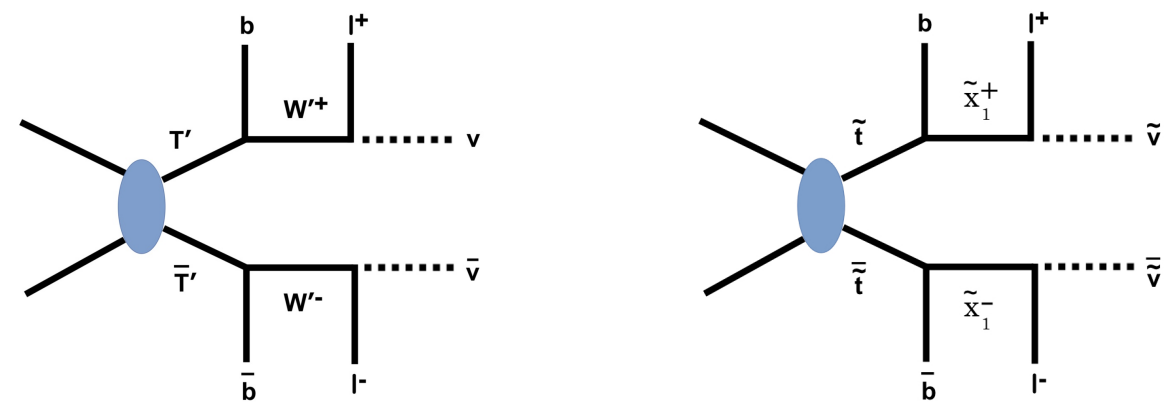

Figure 6. A topology for anything decaying like dilepton top pairs with both a new heavy top partner $\mathrm{T}^{\prime}$ and a new heavy gauge boson $\mathrm{W}^{\prime}$ (left) as well as a pair produced stop quark topology with similar final state (right).

\section{2D mass reconstruction - example topologies}

Several examples topologies for the method are described in this section starting from the benchmark top pairs in the dilepton channel. Next step is a search for anything decaying like dilepton top pairs, a generic topology concerning heavy top partners. The most generic case concerning massive invisible particles for dark matter searches follows. The 2-Dimensional mass reconstruction can also be applied to other interesting searches with a single unknown mass as well as for a top mass measurement. The identification of dilepton top pairs is another possible application as they constitute the most significant SM background in final states with missing energy.

\subsection{Top pairs}

The method can be tested using existing SM particles such as top quark and W boson in the top pairs dilepton channel (figure 1, left). Simulated samples corresponding to an intergrated luminosity of $50 \mathrm{fb}^{-1}$ for top-pairs and the background processes have been created. The latter consist of single boson (Drell-Yan, W+jets), dibosons (WW, WZ, ZZ) as well as single top samples.

Events were selected by requiring at least two energetic leptons, two energetic jets and missing transverse energy. The selection of leptons required the two highest in $\mathrm{P}_{\mathrm{T}}$, opposite charged electrons or muons in the pseudorapidity region $|\eta|<2.1$. Additionally, the leptons were required to have $\mathrm{P}_{\mathrm{T}}>30 \mathrm{GeV}$ as well as an invariant dilepton mass not close to the $\mathrm{Z}$ boson peak $\left(\mathrm{M}_{l l}<76 \mathrm{GeV}\right.$ and $\left.\mathrm{M}_{l l}>106 \mathrm{GeV}\right)$. The selection of jets required the two highest in transverse momentum jets, with $\mathrm{P}_{\mathrm{T}}>30 \mathrm{GeV}$. The jets were reconstructed by the anti- $\mathrm{k}_{\mathrm{t}}$ algorithm [39] in the pseudorapidity region $|\eta|<2.4$. Finally, events with transverse missing energy less than $100 \mathrm{GeV}$ were rejected.

By applying the method described in the previous section in both signal (top-pairs) and background events, the 2-Dimensional mass distribution presented in figure 4 is created. The top quark mass is shown in figure 5 , using a range of $60-100 \mathrm{GeV}$ for the $\mathrm{W}$ mass. In a similar way, the $\mathrm{W}$ boson mass is presented in figure 5 (right) for a range of $150-200 \mathrm{GeV}$ of 

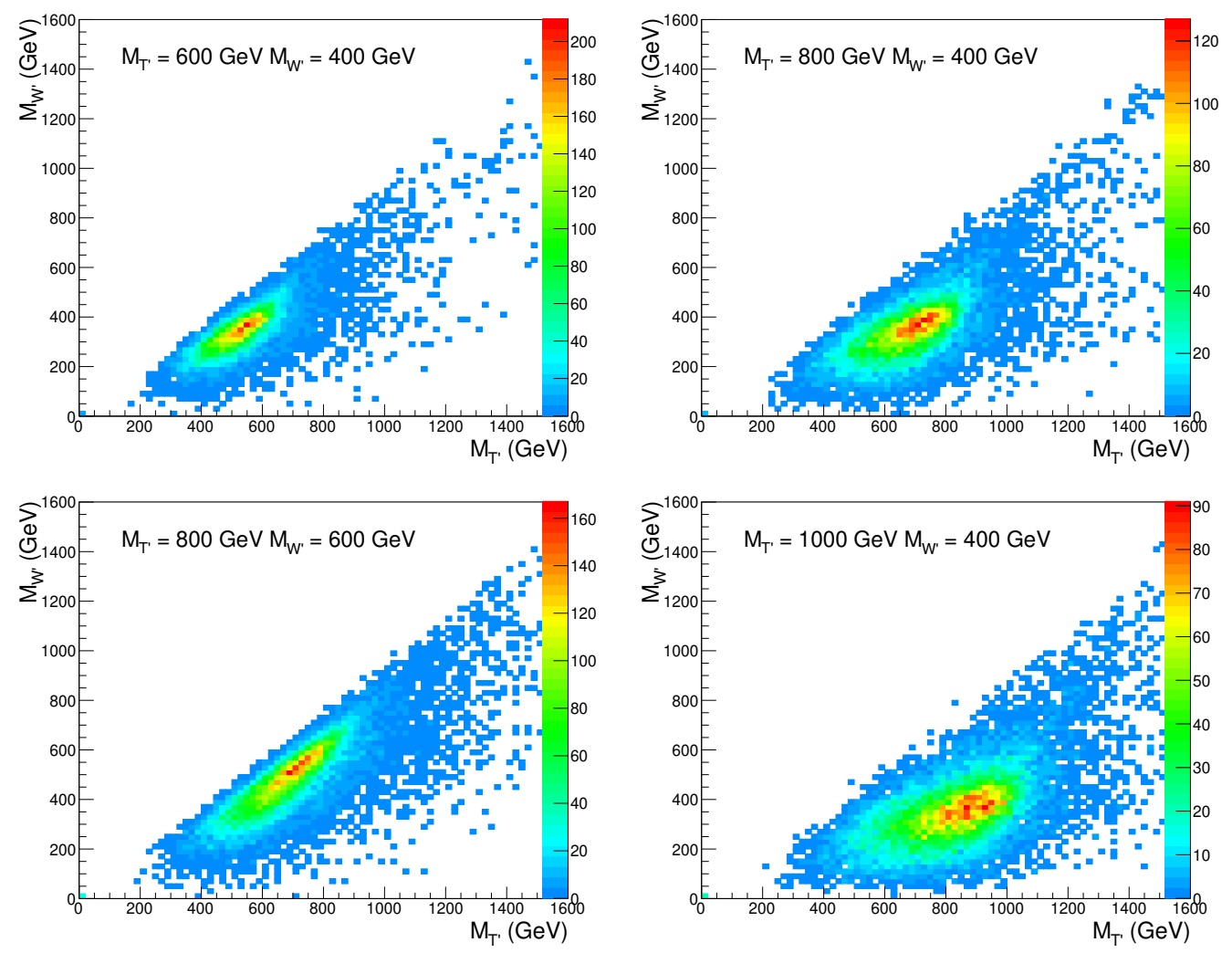

Figure 7. Reconstructed signal distributions (littlest Higgs model) for $\mathrm{M}_{\mathrm{T}^{\prime}}=600 \mathrm{GeV}$, $\mathrm{M}_{\mathrm{W}^{\prime}}=400 \mathrm{GeV} \quad$ (top left), $\mathrm{M}_{\mathrm{T}^{\prime}}=800 \mathrm{GeV}, \quad \mathrm{M}_{\mathrm{W}^{\prime}}=400 \mathrm{GeV}$ (top right), $\mathrm{M}_{\mathrm{T}^{\prime}}=800 \mathrm{GeV}$, $\mathrm{M}_{\mathrm{W}^{\prime}}=600 \mathrm{GeV}$ (bottom left) as well as $\mathrm{M}_{\mathrm{T}^{\prime}}=1000 \mathrm{GeV}, \mathrm{M}_{\mathrm{W}^{\prime}}=400 \mathrm{GeV}$ (bottom right).

the top quark mass. It is worth mentioning that the $\mathrm{W}$ boson distribution has a resonance shape for a leptonic $\mathrm{W}$ decay.

So without any a priori knowledge of their masses or of the underlying theory, both top quark and $\mathrm{W}$ boson can be observed simultaneously by assuming only the event topology. This is a proof of principle for the method, which can then be applied to searches for new hypothetical particles with unknown masses.

\subsection{Search for anything decaying like dilepton top pairs}

The next step is the most generic search for anything decaying like dilepton top pairs ( $\mathrm{pp} \rightarrow \mathrm{T}^{\prime} \overline{\mathrm{T}}^{\prime} \rightarrow \mathrm{W}^{\prime} \mathrm{bW}^{\prime} \mathrm{b}$ ), in their dilepton final state in which both $\mathrm{W}^{\prime}$ bosons decay leptonically ( $\mathrm{W}^{\prime} \rightarrow \mathrm{e} / \mu, \nu$ ). More specifically, this topology has two unknown particles, a new heavy top partner $\mathrm{T}^{\prime}$ and a new heavy charged gauge boson $\mathrm{W}^{\prime}$ (figure 6 , left). It is a search for a heavy top partner in a quite generic topology. The signal samples as already mentioned are based on the littlest Higgs model.

The selection is similar with the one described in section 4.1: the two highest in $\mathrm{P}_{\mathrm{T}}$ opposite charged electrons or muons were selected. The leptons were required to have $\mathrm{P}_{\mathrm{T}}>30 \mathrm{GeV}$, in the pseudorapidity region $|\eta|<2.1$. Additionally, the dilepton invariant mass should not be close to the $\mathrm{Z}$ boson peak $\left(\mathrm{M}_{l l}<76 \mathrm{GeV}\right.$ and $\left.\mathrm{M}_{l l}>106 \mathrm{GeV}\right)$. The 


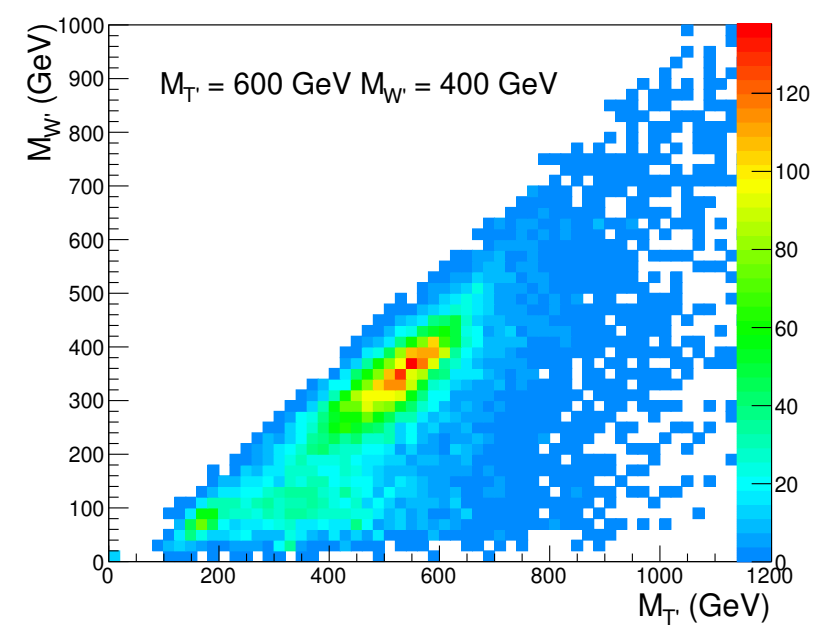

Figure 8. Reconstructed signal distributions for $\mathrm{M}_{\mathrm{T}^{\prime}}=600 \mathrm{GeV}, \mathrm{M}_{\mathrm{W}^{\prime}}=400 \mathrm{GeV}$ (littlest Higgs model) together with the expected background processes corresponding to an intergrated luminosity of $50 \mathrm{fb}^{-1}$ at $13 \mathrm{TeV}$.

two highest in transverse momentum jets were selected, with $\mathrm{P}_{\mathrm{T}}>30 \mathrm{GeV}$ in the pseudorapidity region $|\eta|<2.4$. Finally, events with transverse missing energy less than $200 \mathrm{GeV}$ were rejected.

The performance of the 2-Dimensional mass reconstruction can be seen in figure 7 for several signal samples. Reconstructed signal together with the background procceses are presented in figure 8 . The lower region of the 2-Dimensional mass plane is populated by top pairs. Signal and background events live in different regions of the mass plane, resulting in a good discrimination between them. The invariant mass of the $T^{\prime} \overline{\mathrm{T}}^{\prime}$ system is an interesting observable to monitor for possible new heavy neutral gauge bosons (e.g $\left.\mathrm{pp} \rightarrow \mathrm{Z}^{\prime} \rightarrow \mathrm{T}^{\prime} \overline{\mathrm{T}}^{\prime}\right)$

\subsection{Search for stop pairs}

The most generic case shown in figure 1 (right) concerns massive invisible particles. This scenario is interesting as such topologies are predicted by BSM models that include the lightest supersymmetric particle (LSP) as a dark matter candidate. Such a case that can be searched at the LHC is the pair produced supersymmetric top quark shown in figure 6 (right). In this specific channel the stop decays to the lightest chargino and $\mathrm{b}$ quark, with the chargino decaying further to a lepton and an sneutrino $\left(\mathrm{pp} \rightarrow \tilde{\tilde{\mathrm{t}}} \rightarrow \mathrm{x}_{1}^{+} \mathrm{bx}_{1}^{-} \overline{\mathrm{b}} \rightarrow \mathrm{bl}^{+} \tilde{\nu} \overline{\mathrm{bl}} \mathrm{l}^{-} \overline{\tilde{\nu}}\right)$. The final state is similar to dilepton top pairs consisting of two leptons and bjets as well as large missing energy.

There is an extra unknown mass with respect to the previous cases as the neutrino is replaced by a massive particle. The analytical solution can still provide the correct momenta for the massive sneutrino, given the masses of the other particles as described in [31]. For the 2-Dimensional mass reconstruction, one option is to set the extra unknown mass to zero and perform the method as described in section 3. The event selection applied for signal and SM background processes is the same as the one described in section 4.2. 

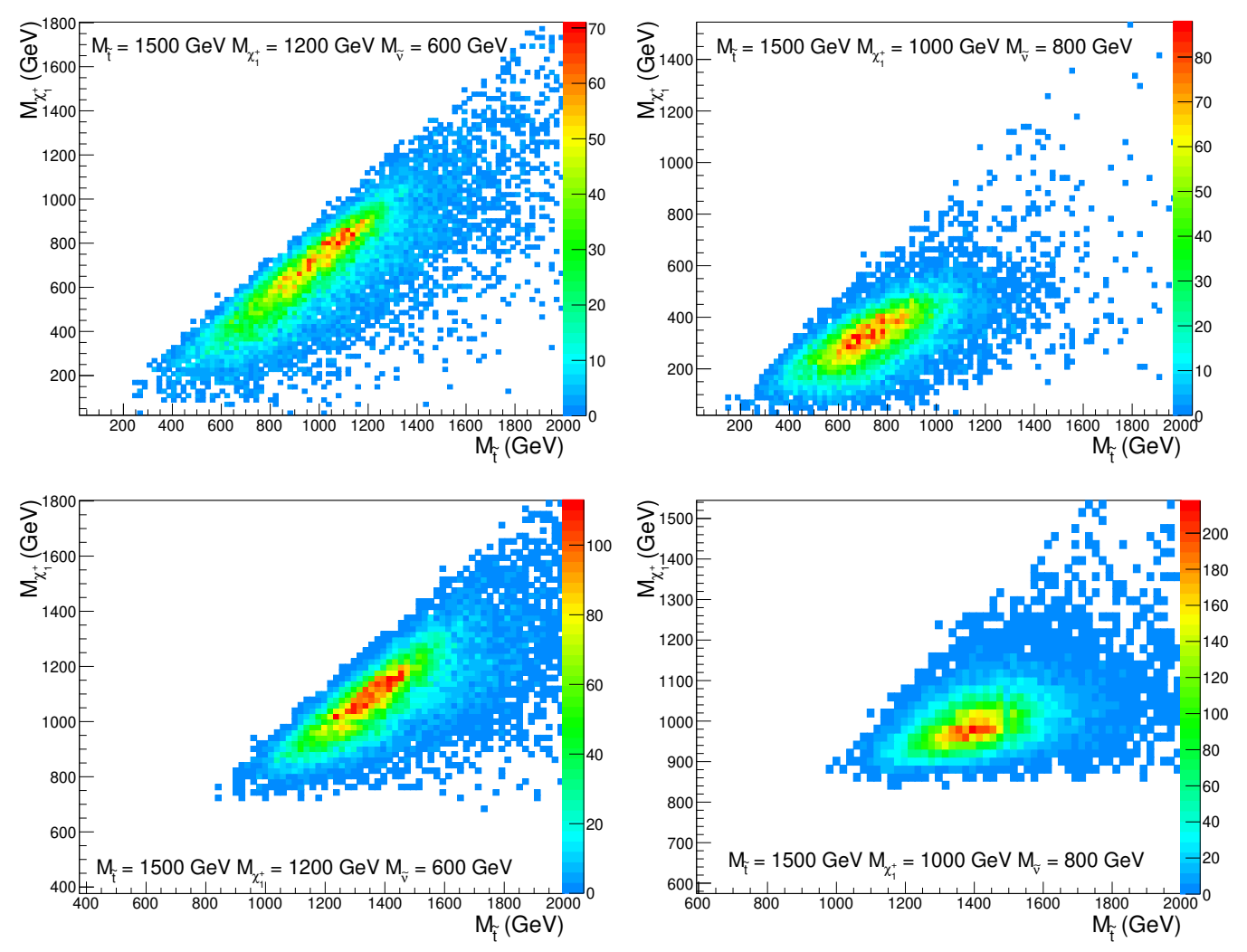

Figure 9. Reconstructed signal distributions assuming zero $\mathrm{M}_{\tilde{\nu}}$ for generated $\mathrm{M}_{\tilde{\mathrm{t}}}=1500 \mathrm{GeV}$, $\mathrm{M}_{\mathrm{x}_{1}^{+}}=1200 \mathrm{GeV}, \mathrm{M}_{\tilde{\nu}}=600 \mathrm{GeV}$ (top left) and $\mathrm{M}_{\tilde{\mathrm{t}}}=1500 \mathrm{GeV}, \mathrm{M}_{\mathrm{x}_{1}^{+}}=1000 \mathrm{GeV}, \mathrm{M}_{\tilde{\nu}}=800 \mathrm{GeV}$ (top right). On the bottom plots the same signal distributions reconstructed assuming nonzero (generated) $\mathrm{M}_{\tilde{\nu}}$ for $\mathrm{M}_{\tilde{\mathrm{t}}}=1500 \mathrm{GeV}, \mathrm{M}_{\mathrm{x}_{1}^{+}}=1200 \mathrm{GeV}, \mathrm{M}_{\tilde{\nu}}=600 \mathrm{GeV}$ (bottom left) and $\mathrm{M}_{\tilde{\mathrm{t}}}=1500 \mathrm{GeV}, \mathrm{M}_{\mathrm{x}_{1}^{+}}=1000 \mathrm{GeV}, \mathrm{M}_{\tilde{\nu}}=800 \mathrm{GeV}$ (bottom right).

The results for two different signal masses can be seen in figure 9 (top). A peak in the 2-Dimensional mass plane is reconstructed as in previous cases, displaced to lower values. Signal together with the backround processes (mainly top pairs) can be seen in figure 10 . The most important conclusion is that in terms of discovery, it is still a bump hunt in the 2-Dimensional plane with signal and background rather well separated and living in different regions of the plane. Another important point is the fact that there is a single entry per event in the 2-Dimensional mass distribution resulting in a small background contamination from the steeply falling spectrum into the signal region. So one can search for dark matter candidates at the LHC by bump hunting instead of missing energy related observables or kinematic endpoints.

In case dark matter exist in the form of a WIMP and is within LHC reach, the following path might be feasible: first use the 2-Dimensional mass reconstruction as described above to take advantage of the bump hunting with a single entry per event for as fast discovery as possible. Bump hunting offers a robust technique with strong signal and background discrimination. The single entry per event offers the possibility for a small background contamination into the signal region. Then, after discovery, use a complementary method 


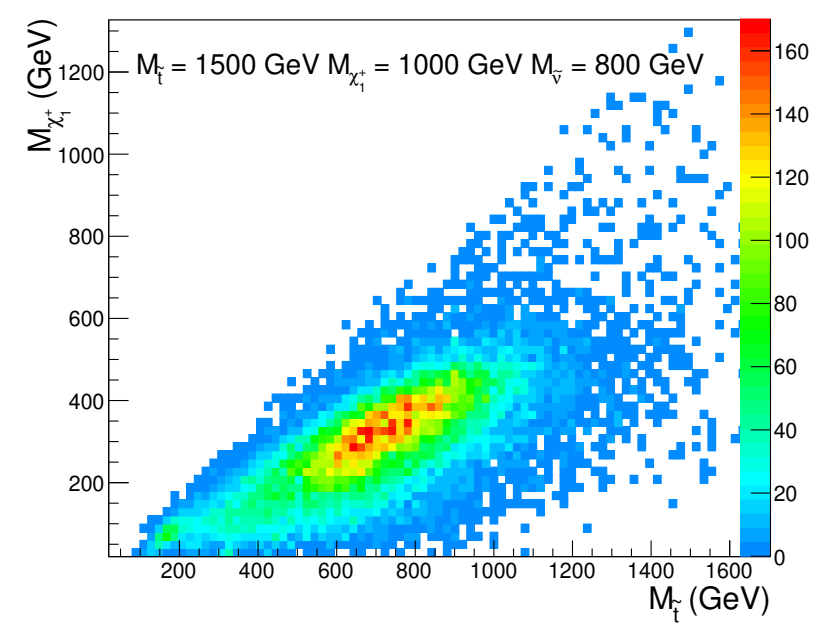

Figure 10. Reconstructed signal distributions for stop pair production with $\mathrm{M}_{\tilde{\mathrm{t}}}=1500 \mathrm{GeV}$, $\mathrm{M}_{\mathrm{x}_{1}^{+}}=1000 \mathrm{GeV}, \mathrm{M}_{\tilde{\nu}}=800 \mathrm{GeV}$ together with the expected background processes corresponding to an intergrated luminosity of $50 \mathrm{fb}^{-1}$ at $13 \mathrm{TeV}$.

to measure the third mass as described for example in [40]. The 2-Dimensional mass reconstruction can be performed once more with the estimated third mass as input, moving the reconstructed masses close to their real values (figure 9, bottom). So for our understanding of the BSM physics after discovery, the method can also provide two of its parameters (e.g $\mathrm{M}_{\tilde{\mathrm{t}}}, \mathrm{M}_{\mathrm{x}_{1}}$ ), with respect to a third one (e.g $\mathrm{M}_{\tilde{\nu}}$ ).

\subsection{Top pair identification/other topologies}

A possible application of the 2-Dimensional mass reconstruction is the identification of dilepton top pair events. This is the most significant SM background for searches performed in final states with missing energy, as it populates the tail of the missing energy related observables used for discovery. This is the same region where possible signal events might exist. The 2-Dimensional mass plane allows the significant suppression of dilepton top pairs by imposing constraints on the masses $\mathrm{M}_{\mathrm{T}}$ and $\mathrm{M}_{\mathrm{W}}$.

Other topologies with a single unknown mass can be reconstructed using the same method. The classic search for a heavy top partner decaying as the SM top quark is such an example ( $\left.\mathrm{pp} \rightarrow \mathrm{T}^{\prime} \overline{\mathrm{T}}^{\prime} \rightarrow \mathrm{WbWb}\right)$. Another applicable topology is the search for a new heavy neutral gauge boson decaying to SM top pairs ( $\mathrm{pp} \rightarrow \mathrm{Z}^{\prime} \rightarrow \mathrm{t} \overline{\mathrm{t}}$ ).

\section{Mass measurements}

After a possible discovery, the next step will be to measure the masses of the new particles. One option is to project the 2-Dimensional distributions to the mass axis, making the problem similar to 1-Dimensional cases such as the top quark measurement. All the relevant experience and the methodology developed for the latter can be exploited in this scenario. The known effects that have an impact on the reconstructed top quark masses are likely to be relevant also in the 2-Dimensional case. In addition, the extra mass available in the 2-Dimensional case can be exploited in the effort to constrain systematic effects. 
So, the existing SM top pairs are a helpful tool and a guide for the mass measurements, as in the case of discovery. The dilepton final state is the cleanest as far as the background is concerned when compared to the semileptonic and hadronic cases. It is considered more challenging in terms of the estimation of the dominant jet energy scale systematic effect. This is due to the fact that both hadronic and semileptonic channels allow the simultaneous reconctruction of the $\mathrm{W}$ boson mass, which can further be used for the estimation of the jet energy scale uncertainty. The 2-Dimensional mass reconstruction offers the opportunity to have both masses $\mathrm{M}_{\mathrm{T}}, \mathrm{M}_{\mathrm{W}}$ simultaneously reconstructed in the relatively clean in terms of background dilepton channel, for a competitive top mass measurement.

After a possible discovery of new particles, the mass measurements is the next step towards understanding the nature of the new physics. The masses constitute basic parameters and can constrain the new physics to BSM models compatible with the discovery. As already mentioned, one possible method is to project the 2-Dimensional distribution to both x-y mass axis. Several templates in small steps of the mass axis can be produced to find which one fits best the data. The fit could be performed simultaneously in both projected masses. By including the low mass region in the fit, a control region is available for constraining the systematic effects. More specifically, the region of top quark and $\mathrm{W}$ boson mass is affected by the jet energy scale/resolution, btagging efficiency, missing energy scale in a similar way as the signal region. Any shift in the jet energy scale will be reflected in both $\mathrm{W}$ boson and top quark mass distributions. Thus, by including the low mass region in the fit, our good knowledge of top quark and W boson mass can be exploitted to constrain the nuisance parameters of the measurement.

The possible effects that are important for the 2-Dimensional mass reconstruction can be further studied by simulation. The latter has several steps: the generation of the hard proccess, parton showers, hadronization, as well as the detector response. All these steps can be checked for their impact on the final reconstructed masses. In figure 9 (bottom) the masses are reconstructed slightly lower than their generated values. In order to check the effect of each step, the 2-Dimensional mass reconstruction is applied to different stages of the simulation process.

Initially, the partons of the hard process are used as an input to the method. This stage does not include hadronization, initial/final state radiation, jet clustering and the detector response. The two highest in transverse momentum quarks and leptons with $\mathrm{P}_{\mathrm{T}}>30 \mathrm{GeV}$ are chosen, with missing energy originating from both neutrino and neutralinos. The result of the 2-Dimensional reconstruction using the partons of the hard process can be seen in figure 11. The small shift to lower masses observed in figure 9 (right bottom) is not visible in this case.

In the next step, the generated particles and jets after the hardonization process performed by Pythia8 are used as an input to the 2-Dimensional mass reconstruction. Generated jets are clustered from generator level long-lived particles obtained after hadronization. The two highest in transverse momenta generated jets and leptons (electrons or muons) having a $\mathrm{P}_{\mathrm{T}}>30 \mathrm{GeV}$ are chosen. In order to check the effect of initial/final state radiation (ISR/FSR), the procedure has been performed twice, switching on/off the ISR/FSR. 


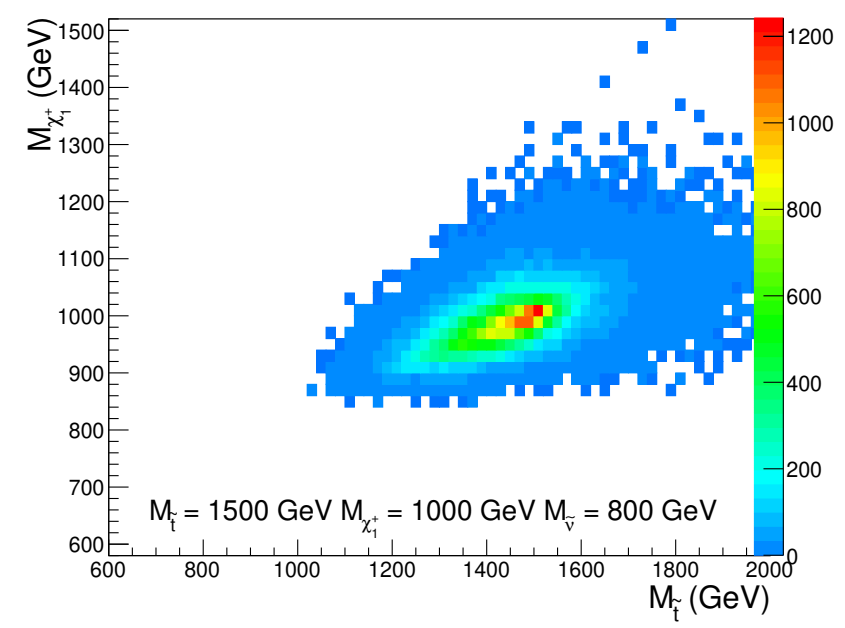

Figure 11. The results of the 2-Dimensional mass reconstruction of stop quark and chargino when using the partons of the hard process as an input. The events have been generated for $\mathrm{M}_{\tilde{\mathrm{t}}}=1500 \mathrm{GeV}, \mathrm{M}_{\mathrm{x}_{1}^{+}}=1000 \mathrm{GeV}$ and $\mathrm{M}_{\tilde{\nu}}=800 \mathrm{GeV}$.

The results of all steps can be seen in figure 12. In the case of hard process partons, the algorithm reconstructs the masses close to their generated values as mentioned earlier. The masses are slightly shifted to lower values after hadronization and the application of the jet clustering algorithm. The latter, even at the generated level is not perfect and has energy losses (e.g neutrinos). Finally, the detector level objects are shifted a bit more as a result of the detector reconstruction process. It is interesting to notice that these effects are more clear in the case of stop quark mass which has an additional jet compared to the chargino. In a running LHC experiment the shifts of the energy scale are partly being compansated by the jet corrections and are taken into account by the jet energy scale uncertaintly.

Finally, the effect of the width of the new particles to the reconstructed masses has been studied. The results for various widths as a percentage of the mass of both stop quark and chargino can be seen in figure 13.

\section{Conclusions}

Higgs boson search was a bump hunt in an expected more or less region of invariant mass spectrum. The collider, the experiments and the analysis were designed based on accurate simulation predictions. The search for BSM physics is much harder. Well motivated theories with heavy top partners or dark matter candidates predict final states with large missing energy due to invisible particles. In this cases, instead of a bump hunt the search is usually performed in the tail of a missing energy related observable. Not only the shape of signal and background processes are similar, but also the discovery cannot give hints about the nature of new physics.

Mass space is the natural space to search for new particles. Mass observables do not require optimization or training. This paper proposes to search for final states with two invisible particles in the 2-Dimensional mass space of the unknown particles. The 

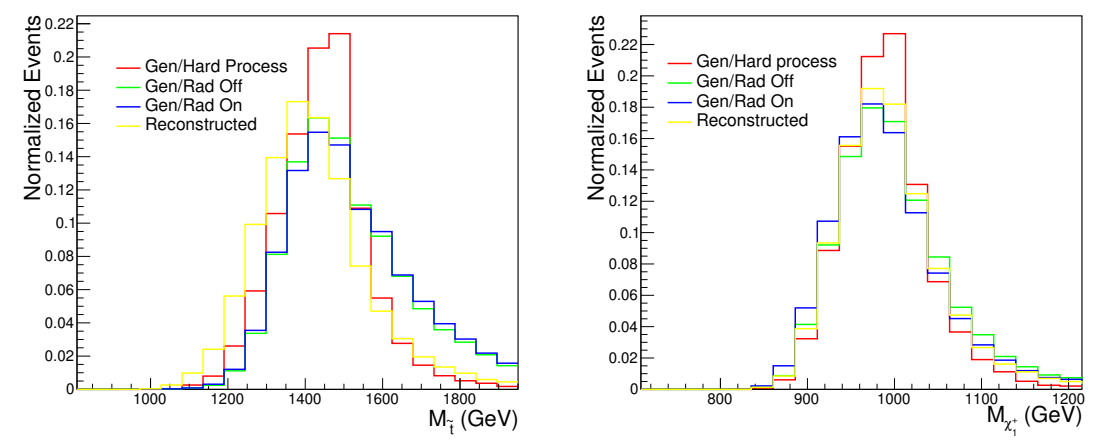

Figure 12. The projected $\mathrm{M}_{\tilde{\mathrm{t}}}$ (left) and $\mathrm{M}_{\mathrm{x}_{1}^{+}}$(right) for both generated and reconstructed level. The generated level is shown for the case the hard process partons are used as input, as well as for generated leptons and jets after hadronization, when the initial/final state radiation is switched Off and On. The events have been generated for $\mathrm{M}_{\tilde{\mathrm{t}}}=1500 \mathrm{GeV}, \mathrm{M}_{\mathrm{x}_{1}^{+}}=1000 \mathrm{GeV}$ and $\mathrm{M}_{\tilde{\nu}}=800 \mathrm{GeV}$.
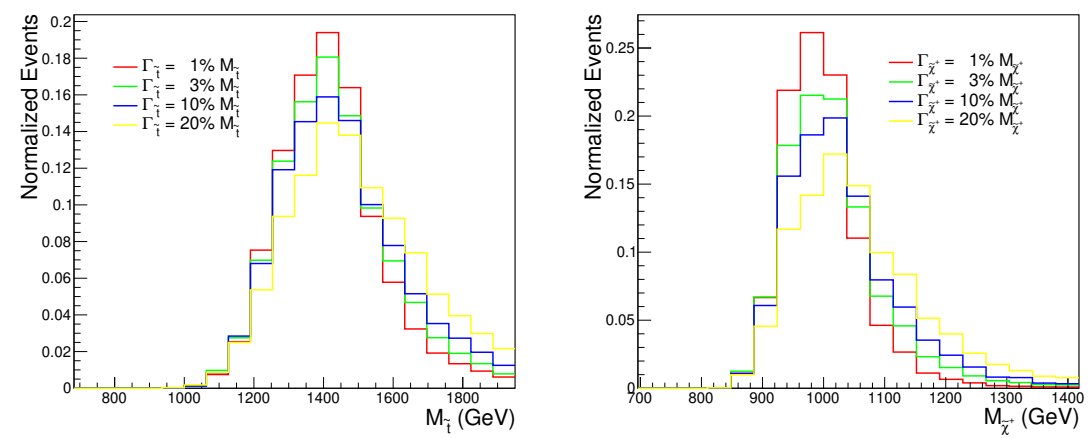

Figure 13. The projected $\mathrm{M}_{\tilde{\mathrm{t}}}$ (left) and $\mathrm{M}_{\mathrm{x}_{1}^{+}}$(right) when the generated width is $1 \%, 3 \%, 10 \%, 20 \%$ of the mass of both stop quark and chargino. The events have been generated for $\mathrm{M}_{\tilde{\mathrm{t}}}=1500 \mathrm{GeV}$, $\mathrm{M}_{\mathrm{x}_{1}^{+}}=1000 \mathrm{GeV}$ and $\mathrm{M}_{\tilde{\nu}}=800 \mathrm{GeV}$.

reconstruction is based on a PDF weight without any matrix elements, to be as model independent as possible for a given topology. Thus, the search is a bump hunt in more than one dimension, making signal discrimination from background processes an easier task. In addition, reconstruction of the unknown masses can give valuable insights to what the new physics might be.

Initially, the proof of principle is presented using the existing SM dilepton top pairs. A generic search for anything decaying like dilepton top pairs with both a new heavy top partner and a new heavy gauge boson is used to show the application of the method in a typical topology with two invisible particles. The most interesting application for dark matter searches at the LHC concerns the topologies with massive invisible particles. The 2Dimensional mass reconstruction in this case allows these searches to be performed as bump hunting with a single entry per event, with all the advantages offered in terms of discovery. Top pair identification is an interesting application for searches using missing energy like observables. The 2-Dimensional mass reconstruction can also be applied to many other topologies with one or two uknown masses as well as for a top mass measurement in the 
dilepton top pairs channel. The method has already been used in CMS Run1 with many interesting topologies awaiting the next LHC Runs.

\section{A System of equations for top pairs in the dilepton channel}

The equations for the top pair system in the dilepton channel are the following:

$$
\begin{aligned}
& \mathrm{m}_{\mathrm{t}}^{2}=\left(\mathrm{E}_{\mathrm{b}}+\mathrm{E}_{\mathrm{l}^{+}}+\mathrm{E}_{\nu}\right)^{2}-\sum_{i=1}^{3}\left(\mathrm{p}_{\mathrm{b}_{\mathrm{i}}}+\mathrm{p}_{\mathrm{l}_{\mathrm{i}}^{+}}+\mathrm{p}_{\nu_{\mathrm{i}}}\right)^{2} \\
& \mathrm{~m}_{\overline{\mathrm{t}}}^{2}=\left(\mathrm{E}_{\overline{\mathrm{b}}}+\mathrm{E}_{\mathrm{l}^{-}}+\mathrm{E}_{\bar{\nu}}\right)^{2}-\sum_{i=1}^{3}\left(\mathrm{p}_{\overline{\mathrm{b}}_{\mathrm{i}}}+\mathrm{p}_{\mathrm{l}_{\mathrm{i}}^{-}}+\mathrm{p}_{\bar{\nu}_{\mathrm{i}}}\right)^{2} \\
& \mathrm{~m}_{\mathrm{W}^{+}}^{2}=\left(\mathrm{E}_{\mathrm{l}^{+}}+\mathrm{E}_{\nu}\right)^{2}-\sum_{i=1}^{3}\left(\mathrm{p}_{\mathrm{l}_{\mathrm{i}}^{+}}+\mathrm{p}_{\nu_{\mathrm{i}}}\right)^{2} \\
& \mathrm{~m}_{\mathrm{W}^{-}}^{2}=\left(\mathrm{E}_{\mathrm{l}^{-}}+\mathrm{E}_{\bar{\nu}}\right)^{2}-\sum_{i=1}^{3}\left(\mathrm{p}_{\mathrm{l}_{\mathrm{i}}^{-}}+\mathrm{p}_{\bar{\nu}_{\mathrm{i}}}\right)^{2} \\
& \mathrm{M}_{\mathrm{ET}_{\mathrm{x}}}=\mathrm{p}_{\nu_{\mathrm{x}}}+\mathrm{p}_{\bar{\nu}_{\mathrm{x}}} \quad \mathrm{E}_{\nu}^{2}=\mathrm{p}_{\nu_{\mathrm{x}}}^{2}+\mathrm{p}_{\nu_{\mathrm{y}}}^{2}+\mathrm{p}_{\nu_{\mathrm{z}}}^{2} \\
& \mathrm{M}_{\mathrm{ET}_{\mathrm{y}}}=\mathrm{p}_{\nu_{\mathrm{y}}}+\mathrm{p}_{\bar{\nu}_{\mathrm{y}}} \quad \mathrm{E}_{\bar{\nu}}^{2}=\mathrm{p}_{\bar{\nu}_{\mathrm{x}}}^{2}+\mathrm{p}_{\bar{\nu}_{\mathrm{y}}}^{2}+\mathrm{p}_{\bar{\nu}_{\overline{\mathrm{z}}}}^{2}
\end{aligned}
$$

Open Access. This article is distributed under the terms of the Creative Commons Attribution License (CC-BY 4.0), which permits any use, distribution and reproduction in any medium, provided the original author(s) and source are credited.

\section{References}

[1] D.J.H. Chung, L.L. Everett, G.L. Kane, S.F. King, J.D. Lykken and L.-T. Wang, The soft supersymmetry breaking Lagrangian: Theory and applications, Phys. Rept. 407 (2005) 1 [hep-ph/0312378] [INSPIRE].

[2] N. Arkani-Hamed, S. Dimopoulos and G.R. Dvali, The hierarchy problem and new dimensions at a millimeter, Phys. Lett. B 429 (1998) 263 [hep-ph/9803315] [INSPIRE].

[3] M. Schmaltz and D. Tucker-Smith, Little Higgs review, Ann. Rev. Nucl. Part. Sci. 55 (2005) 229 [hep-ph/0502182] [INSPIRE].

[4] M. Baak et al., Updated Status of the Global Electroweak Fit and Constraints on New Physics, Eur. Phys. J. C 72 (2012) 2003 [arXiv:1107.0975] [InSPIRE].

[5] H.-C. Cheng, D. Engelhardt, J.F. Gunion, Z. Han and B. McElrath, Accurate Mass Determinations in Decay Chains with Missing Energy, Phys. Rev. Lett. 100 (2008) 252001 [arXiv: 0802.4290] [INSPIRE].

[6] H.-C. Cheng, J.F. Gunion, Z. Han and B. McElrath, Accurate Mass Determinations in Decay Chains with Missing Energy. II, Phys. Rev. D 80 (2009) 035020 [arXiv:0905.1344] [INSPIRE].

[7] G. Anagnostou, Model Independent Search in 2-Dimensional Mass Space, EPJ Web Conf. 71 (2014) 00006 [arXiv:1112.3379] [inSPIRE]. 
[8] CMS collaboration, Search in two-dimensional mass space for $\mathrm{T}^{\prime} \mathrm{T}^{\prime} \rightarrow \mathrm{W}^{\prime} \mathrm{bW}^{\prime} \mathrm{b}$ in the dilepton final state in proton-proton collisions at $8 \mathrm{TeV}$, Tech. Rep. CMS-PAS-B2G-12-025, CERN, Geneva (2015).

[9] S.Beranek, Model independent search for new particles in two-dimensional mass space using events with missing energy, two jets and two leptons with the CMS detector, CERN-THESIS-2017-115 (2017).

[10] CMS collaboration, Beyond two generations, EPJ Web Conf. 126 (2016) 04001 [InSPIRE].

[11] G. Anagnostou, Searching in 2-D Mass Space For Final States with 2 Invisible Particles, talk in Dark Matter Identification, Connecting Theory and Signature Space conference, Mainz, Germany, April 2019, https://indico.mitp.uni-mainz.de/event/180/timetable/\#20190405.

[12] T. Sjöstrand, S. Mrenna and P.Z. Skands, A Brief Introduction to PYTHIA 8.1, Comput. Phys. Commun. 178 (2008) 852 [arXiv:0710.3820] [inSPIRE].

[13] T. Han, H.E. Logan, B. McElrath and L.-T. Wang, Phenomenology of the little Higgs model, Phys. Rev. D 67 (2003) 095004 [hep-ph/0301040] [INSPIRE].

[14] J. Reuter, M. Tonini and M. de Vries, Little Higgs Model Limits from LHC - Input for Snowmass 2013, in Snowmass 2013: Workshop on Energy Frontier, (2013) [arXiv: 1307.5010] [INSPIRE].

[15] W. Kilian, T. Ohl and J. Reuter, WHIZARD: Simulating Multi-Particle Processes at LHC and ILC, Eur. Phys. J. C 71 (2011) 1742 [arXiv:0708.4233] [INSPIRE].

[16] M. Moretti, T. Ohl and J. Reuter, O'Mega: An optimizing matrix element generator, hep-ph/0102195 [INSPIRE].

[17] J. Alwall et al., A Les Houches Interface for BSM Generators, FERMILAB-CONF-07-621-T [arXiv:0712.3311] [INSPIRE].

[18] J. Alwall et al., The automated computation of tree-level and next-to-leading order differential cross sections, and their matching to parton shower simulations, JHEP 07 (2014) 079 [arXiv: 1405.0301] [INSPIRE].

[19] Y. Li and F. Petriello, Combining QCD and electroweak corrections to dilepton production in FEWZ, Phys. Rev. D 86 (2012) 094034 [arXiv: 1208.5967] [InSPIRE].

[20] J.M. Campbell, R.K. Ellis and C. Williams, Vector boson pair production at the LHC, JHEP 07 (2011) 018 [arXiv: 1105.0020] [InSPIRE].

[21] T. Gehrmann et al., $W^{+} W^{-}$Production at Hadron Colliders in Next to Next to Leading Order QCD, Phys. Rev. Lett. 113 (2014) 212001 [arXiv:1408.5243] [INSPIRE].

[22] M. Cacciari, M. Czakon, M. Mangano, A. Mitov and P. Nason, Top-pair production at hadron colliders with next-to-next-to-leading logarithmic soft-gluon resummation, Phys. Lett. $B \mathbf{7 1 0}$ (2012) 612 [arXiv:1111.5869] [INSPIRE].

[23] P. Bärnreuther, M. Czakon and A. Mitov, Percent Level Precision Physics at the Tevatron: First Genuine NNLO QCD Corrections to $q \bar{q} \rightarrow t \bar{t}+X$, Phys. Rev. Lett. 109 (2012) 132001 [arXiv: 1204.5201] [INSPIRE].

[24] M. Czakon and A. Mitov, NNLO corrections to top-pair production at hadron colliders: the all-fermionic scattering channels, JHEP 12 (2012) 054 [arXiv:1207.0236] [INSPIRE].

[25] M. Czakon and A. Mitov, NNLO corrections to top pair production at hadron colliders: the quark-gluon reaction, JHEP 01 (2013) 080 [arXiv:1210.6832] [INSPIRE]. 
[26] M. Beneke, P. Falgari, S. Klein and C. Schwinn, Hadronic top-quark pair production with NNLL threshold resummation, Nucl. Phys. B 855 (2012) 695 [arXiv:1109.1536] [InSPIRE].

[27] M. Czakon, P. Fiedler and A. Mitov, Total Top-Quark Pair-Production Cross Section at Hadron Colliders Through $O\left(\alpha_{S}^{4}\right)$, Phys. Rev. Lett. 110 (2013) 252004 [arXiv:1303.6254] [INSPIRE].

[28] M. Czakon and A. Mitov, Top++: A program for the Calculation of the Top-Pair Cross-Section at Hadron Colliders, Comput. Phys. Commun. 185 (2014) 2930 [arXiv:1112.5675] [INSPIRE].

[29] DELPHES 3 collaboration, DELPHES 3, A modular framework for fast simulation of a generic collider experiment, JHEP 02 (2014) 057 [arXiv:1307.6346] [INSPIRE].

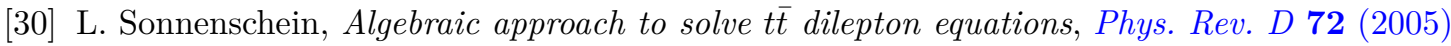
095020 [hep-ph/0510100] [INSPIRE].

[31] L. Sonnenschein, Analytical solution of ttbar dilepton equations, Phys. Rev. D 73 (2006) 054015 [Erratum ibid. 78 (2008) 079902] [hep-ph/0603011] [INSPIRE].

[32] M. Guzzi, P. Nadolsky, E. Berger, H.-L. Lai, F. Olness and C.P. Yuan, CT10 parton distributions and other developments in the global QCD analysis, arXiv:1101.0561 [INSPIRE].

[33] A. Buckley et al., LHAPDF6: parton density access in the LHC precision era, Eur. Phys. J. C 75 (2015) 132 [arXiv: 1412.7420] [INSPIRE].

[34] K. Kondo, Dynamical Likelihood Method for Reconstruction of Events with Missing Momentum I, Method and Toy Models, J. Phys. Soc. Jap. 57 (1988) 4126.

[35] K. Kondo, Dynamical Likelihood Method for Reconstruction of Events with Missing Momentum II, Mass Spectra for $2 \rightarrow 2$ Processes, J. Phys. Soc. Jap. 60 (1991) 836.

[36] R.H. Dalitz and G.R. Goldstein, The decay and polarization properties of the top quark, Phys. Rev. D 45 (1992) 1531 [INSPIRE].

[37] D0 collaboration, Measurement of the Top Quark Mass in Final States with Two Leptons, Phys. Rev. D 80 (2009) 092006 [arXiv:0904.3195] [INSPIRE].

[38] CMS collaboration, Measurement of the Top-Quark Mass in $t \bar{t}$ Events with Dilepton Final States in pp Collisions at $\sqrt{s}=7 \mathrm{TeV}$, Eur. Phys. J. C 72 (2012) 2202 [arXiv:1209.2393] [INSPIRE].

[39] M. Cacciari, G.P. Salam and G. Soyez, The anti- $k_{t}$ jet clustering algorithm, JHEP 04 (2008) 063 [arXiv: 0802.1189] [INSPIRE].

[40] D. Kim, K.T. Matchev and P. Shyamsundar, Kinematic Focus Point Method for Particle Mass Measurements in Missing Energy Events, JHEP 10 (2019) 154 [arXiv:1906.02821] [INSPIRE]. 